\title{
Electrochemotherapy guided by intraoperative fluorescence imaging for the treatment of inoperable peritoneal micro-metastases
}

\author{
V. Josserand, ${ }^{\mathrm{a}, \mathrm{b}, 1}$ M. Kéramidas, ${ }^{\mathrm{a}, \mathrm{b}, 1}{ }^{1}$ J. Lavaud,,${ }^{\mathrm{a}, \mathrm{b}}$ C. Righini, ${ }^{\mathrm{a}, \mathrm{b}, \mathrm{c}}{ }^{\mathrm{J}}$. Vollaire, ${ }^{\mathrm{a}, \mathrm{b}}$ E. Bellard, ${ }^{\mathrm{d}, \mathrm{e}}$ M.P. Rols, ${ }^{\mathrm{d}, \mathrm{e}}$ \\ J. Teissié, ${ }^{\mathrm{d}, \mathrm{e}}$ J.L. Coll, ${ }^{\mathrm{a}, \mathrm{b}, *}$ M. Golzio d, e, ** \\ a INSERM U1209, Institut Albert Bonniot, F-38000 Grenoble, France \\ ${ }^{\mathrm{b}}$ Univ. Grenoble Alpes, Institut Albert Bonniot, F-38000 Grenoble, France \\ ${ }^{c}$ CHU, Grenoble, France \\ d CNRS, IPBS, Toulouse, France \\ e Université de Toulouse, UPS, IPBS, Toulouse, France
}

\section{A R T I C L E I N F O}

\section{Article history:}

Received 8 February 2016

Accepted 1 May 2016

Available online $\mathrm{xxx}$

\section{Keywords:}

Electroporation

Electrochemotherapy

Peritoneal metastases

Optical imaging

Fluorescence guided surgery
Received in revised form 25 April 2016

Tumor targeting

\section{A B S T R A C T}

Surgery is often the first therapeutic indication in cancer. Patient survival essentially depends on the completeness of tumor resection. This is a major challenge, particularly in patients with peritoneal carcinomatosis (PC), where tumors are widely disseminated in the large peritoneal cavity. These small tumors can be difficult to visualize and are often positioned in delicate locations, further increasing the risk of producing serious tissue/organ damage during their ablation. We propose an innovative therapeutic approach based on intraoperative fluorescence (IF) guided electrochemotherapy (ECT) for the treatment of peritoneal micro-metastases. ECT combines the effects of tissue electro-permeabilization (EP) with the administration of an antimitotic agent (bleomycin) that has poor permeability across intact membranes. IF significantly improves the detection of small tumor lesions. ECT is clinically validated for the treatment of cutaneous tumors in animals and humans, but this is the first time that it has been used along with IF imaging for the targeted treatment of peritoneal metastases in a preclinical model.

We set up a murine model of PC that develops secondarily to the resection of a distant primary tumor. Tumor growth and metastasis were finely monitored by non-invasive multimodal imaging (bioluminescence and 3D fluorescence/microCT). Once metastases were detected, mice were randomized into three groups: the ECT group (bleomycin injected intravenously followed by EP) and 2 control groups (bleomycin alone and EP alone). Twenty four hours after the intravenous injection of the tumor targeting agent Angiostamp ${ }^{\mathrm{TM}} 700$, mice in all groups underwent an abdominal surgery for metastases exploration assisted by fluorescence imaging with the Fluobeam ${ }^{\circledR} 700$ portative device. EP was applied to every nodule detected by IF, except in the bleomycin control group. After surgery, the metastatic invasion was tracked by bioluminescence imaging. In mice treated with bleomycin or EP alone, the metastatic load progressed very rapidly and mice showed no significant difference in lifespan compared to non-operated mice (median lifespan: 27 days vs. 25 days, respectively). In contrast, the mice treated with ECT displayed a decreased metastatic load and an increased survival rate (median lifespan: 34 days). These results provide evidence that IF guided ECT is an effective approach for the treatment of inoperable intraperitoneal micro-metastases.

(C) 2016 Published by Elsevier Ltd.

\section{Introduction}

In oncology, if tumor resection is feasible, surgery is often the first therapeutic option. For peritoneal carcinomatosis from colorectal cancer or recurrent ovarian cancer, a treatment of choice is cytoreductive surgery associated with immediate hyperthermic intraperitoneal chemotherapy. Patient survival essentially depends on the completeness of macroscopic tumor resection and on adjuvant chemotherapy for the elimination of residual tumor nodules smaller

\footnotetext{
* Correspondence to: J.-L. Coll, Institut Albert Bonniot INSERM-UJF U823, 38706 Grenoble, France.

** Correspondence to: M. Golzio, IPBS CNRS, UMR 5089, 205, Route de Narbonne, 31077 Toulouse Cedex, France.

Email addresses: jean-luc.coll@ujf-grenoble.fr (J.L. Coll); muriel.golzio@ipbs.fr (M. Golzio)

1 These authors contributed equally to this work.
}

than $2.5 \mathrm{~mm}$ [1]. Unfortunately, the peritoneal extension, the multifocal spread of the metastases and the close contact with vital organs often lead to incomplete tumor resection. Optimizing the extensiveness of cytoreductive surgery without collateral damage is still a major challenge.

Advances in optical fluorescence imaging, and especially in intraoperative imaging devices associated with the generation of tumor-targeted contrast agents, can improve the normal vision of surgeons [2-6]. In addition to morphological changes and/or discoloration of the tissue that basically guide the surgeon, molecular imaging has the potential to provide additional biological information about the cancerous tissue at molecular and cellular levels [7]. However, despite these improvements in tumor visualization, some cancerous nodules are too dangerous to remove because of their location on sensitive organs or tissues (e.g. arteries, nerves), and cannot be excised without a major risk of causing irreparable damages. Therefore, loco-regional treatments such as cryotherapy, radiofrequency or thermal ablation 
have been developed in combination with surgery [8]. Electrochemotherapy (ECT) has emerged as a new therapy with a high objective response rate (\$_amp_\$gt; 80\%) for treatment of cutaneous and subcutaneous tumors and new ECT protocols are being developed to treat deeper tumors $[9,10]$. ECT bypasses tumor removal and instead consists in the administration (intravenous or intra-tumor injections) of very low doses of non-permeant anticancer drugs, such as bleomycin or cisplatin, followed by short electric pulses applied locally to the tumor. ECT induces an antitumor effect whose main mechanism is the enhanced delivery of these low permeant cytotoxic molecules into tumor cells $[11,12]$. ECT was pioneered for the treatment of equine tumors by the authors [13] and the first clinical trials in human were performed in France by the team of L.M. Mir $[14,15]$. ECT was demonstrated to be a safe procedure since electric pulses used in ECT did not significantly change the expression profile of genes involved in the development of cancer in malignant melanoma cells [16], did not impact drug resistance and did not change the metastatic potential of human melanoma cells [17]. In clinical trials performed in Europe and the USA, ECT has been shown to be well tolerated and effective in the control of primary and metastatic solid tumors of diverse histotypes $[9,10,13,18]$. ECT was described as a safe procedure and is now a routine practice in oncological clinics (National Institute for Health and Clinical Excellence (NICE) guidance (https://www.nice.org.uk/Guidance/IPG446; https://www.nice.org.uk/ Guidance/IPG478). The technological development of new electrodes has allowed for the treatment of deep tumors such as liver and bone metastases, brain tumors, and colorectal and esophageal tumors [9]. ECT was shown to be highly effective, with complete response rates between 60 and $70 \%$ and objective response rate around $80 \%[10,19]$. The overall effectiveness of ECT is also dependent on the involvement of the immune system. Indeed, ECT-mediated tumor regression was dramatically decreased in animals deficient in functional $\mathrm{T}$ lymphocytes, such as immunocompromised nude mice [20]. Nevertheless, ECT being a local treatment, one of the main challenge is to target the electric field onto every tumor nodule.

In the present work, we propose to evaluate, for the first time, an innovative approach of ECT guided by intraoperative fluorescence (IF) imaging for the treatment of inoperable peritoneal micro-metas- tases in a preclinical model. To achieve this aim, we used a micrometastases model in which very aggressive metastases appear secondarily to primary tumor resection. No resection of metastases was performed. Instead, nodules detected by IF imaging were treated by ECT, EP alone or bleomycin alone. This experimental model offers the advantage of being finely controlled, since both primary tumor growth and metastases invasion are monitored over time with noninvasive multimodal imaging (bioluminescence and 3D fluorescence/ microCT).

\section{Material and methods}

\subsection{Cell line and culture conditions}

TSA-pGL3 is a cell line derived from the original TS/Apc mouse adenocarcinoma cell line and stably transfected with the pGL3-luciferase reporter gene (Promega, Charbonnieres, France). Cells were cultured at $37{ }^{\circ} \mathrm{C}$ in a humidified $95 \%$ air $/ 5 \%$ carbon dioxide atmosphere in RPMI 1640 medium supplemented with 1\% glutamine, 10\% fetal bovine serum, 50 units $/ \mathrm{mL}$ penicillin, $50 \mu \mathrm{g} / \mathrm{mL}$ streptomycin and $700 \mu \mathrm{g} / \mathrm{mL}$ Geneticin (G418 sulphate; Gibco, Paisley, UK). These cells express low levels of integrin $\alpha \mathrm{v} \beta 3$ [21].

\subsection{Animal and tumor models}

All animal experiments were conducted in agreement with the Principles of Laboratory Animal Care (National Institutes of Health publication no. 86-23, revised 1985) and approved by the regional ethics committee. The animal model setup is summarized in Fig. 1.

Female 8 weeks old balb/c mice (Janvier, Le Genest-Isle, France) were anesthetized (isoflurane/oxygen $3.5 \%$ for induction and $1.5 \%$ thereafter) and shaved, then a $2 \mathrm{~cm}$ incision was made in the left flank so that the kidney could be slightly pushed out of the cavity. By using a Hamilton syringe, $15 \times 10^{3}$ TSA-pGL3 cells, suspended in $20 \mu \mathrm{L}$ phosphate-buffered saline, were injected under the renal capsule. Seven days later (day 8), the tumor-bearing kidney was surgically removed. After each surgery, an antibiotherapy (sulfamethoxazole $1 \mathrm{~g} / \mathrm{L}$, tetracycline $1 \mathrm{~g} / \mathrm{L}$, trimethoprim $200 \mathrm{mg} / \mathrm{L}$ ) was admin-

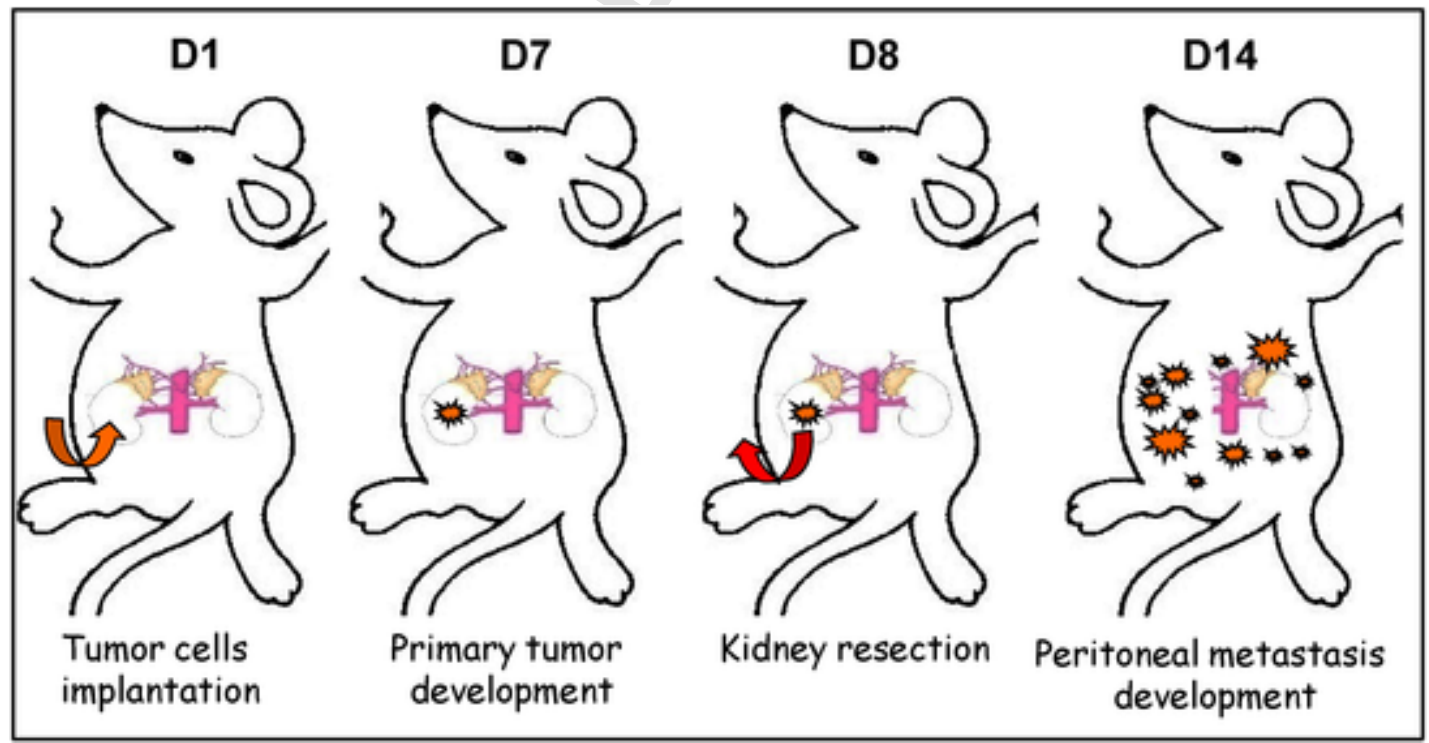

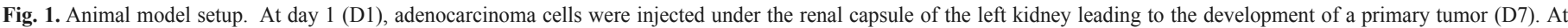
day 8 (D8), the tumor-bearing kidney was surgically removed. At day 14 (D14), small scattered nodules resembling peritoneal carcinomatosis appeared in the peritoneal cavity. 
istered in drinking water for 4 days. Six days after the removal of the tumor-bearing kidney (day 14), peritoneal carcinomatosis was monitored by in vivo bioluminescence imaging.

\subsection{Bioluminescence imaging}

Primary tumor growth and metastases development were monitored by in vivo bioluminescence imaging 6 days after tumor cell injection (D7), 6 days after tumor kidney removal (D14) and then twice a week until death. Five minutes before imaging, mice received an intraperitoneal injection of $150 \mu \mathrm{g} / \mathrm{g}$ of D-luciferin (Promega, France) and then were anesthetized (isoflurane $4 \%$ for induction and $1.5 \%$ thereafter) and placed in the optical imaging system (Aequoria, Hamamatsu Photonics, France). This allowed localization and quantitation of luciferase-positive tumor cells before and after treatment. Semiquantitative data were obtained from the bioluminescence images by drawing regions of interest on the abdomen with the Wasabi software (Hamamatsu Photonics, France). Results were expressed as a number of relative light units per pixel per second (RLU/pix/s) and the evolution of metastases after treatment was expressed as a percentage relative to the measure at day 14 .

\subsection{Fluorescence tomography/microCT imaging}

For tumor and metastases imaging via integrin $\alpha v \beta 3$ targeting [3], Angiostamp ${ }^{\mathrm{TM}} 700$ (Fluoptics, France) was injected intravenously (10 nmol, $200 \mu \mathrm{L}$ ) at day 7 (prior to kidney resection), and at day 14 (the day before treatment). Four hours later, mice were injected intravenously with $100 \mu \mathrm{L}$ of Visipac $320 \mathrm{mg} / \mathrm{mL}$ iodine (GE Healthcare, USA) and were placed in a mobile animal holder compatible with the previously described fDOT (CEA-LETI, France) for 3D fluorescence imaging [21] and the microCT scanner (ScancoMedical, Switzerland) for whole body anatomical imaging. MicroCT acquisitions were made at $45 \mathrm{keV}$ with a $177 \mu \mathrm{A}$ intensity, a $300 \mathrm{~ms}$ integration time and a $80 \mu \mathrm{m}$ isotropic voxel size. Fluorescence scanning was performed on the abdominal region only. MicroCT and 3D fluorescence data were reconstructed separately by the respective software systems and $3 \mathrm{D}$ images were superimposed for bimodal visualization with the open source image processing program Image $\mathrm{J}(\mathrm{NIH})$.

\subsection{Intraoperative fluorescence imaging}

On the day of the surgery (day 15), tracking of metastases was performed with the previously described handheld NIR 2D-Fluorescence Reflectance Imaging device (Fluobeam ${ }^{\circledR} 700$, Fluoptics, France) $[3,4]$. This system offers real time fluorescence imaging in a bright and open field environment for surgery. The surgeon can therefore operate as usual but with the assistance of a screen that dis- plays the location of fluorescent tumors. In order to evaluate the Fluobeam ${ }^{\circledR} 700$ sensitivity, a standard curve was prepared from serial Angiostamp ${ }^{\mathrm{TM}} 700$ dilutions. A signal-to-noise ratio of 1.5 or greater was considered the positive signal threshold. Under these conditions, a positive signal could be detected on a $10 \mu \mathrm{L}$ aliquot containing $0.5 \mathrm{nmol} / \mathrm{L}$ of probe $(0.5 \mathrm{fmol} / \mu \mathrm{L})$ with a $100 \mathrm{~ms}$ exposure (data not shown).

\subsection{Electrochemotherapy}

When metastases were detected by bioluminescence imaging (day $14)$, mice were randomized into 3 groups ( $n=12$ mice/group) - G1: ECT group (bleomycin injected intravenously followed by electric field application) G2: bleomycin alone (control) and G3: EP alone (control).

At day 15, mice from $\mathrm{G} 1$ and $\mathrm{G} 2$ received an intravenous injection of a low concentration of bleomycin $(5 \mathrm{mg} / \mathrm{kg}$ in $0.15 \mathrm{M} \mathrm{NaCl})$ whereas $\mathrm{G} 3$ received $0.15 \mathrm{M} \mathrm{NaCl}$ only. Five minutes later, mice were anesthetized and underwent an abdominal surgery for image-guided metastases tracking. For G1 and G3, each metastasis observed was immediately subjected to EP. Electric parameters were 8 pulses $(100 \mu \mathrm{s}$ at $10 \mathrm{~Hz}$ frequency) delivered with stainless steel non-invasive contact electrodes $(1 \mathrm{~mm})$ connected to a S20 generator from Betatech (St Orens, France). The amplitude was 110 or $130 \mathrm{~V}$. These electrodes were especially designed for this study (Fig. 2B and C) and could be moved easily inside the peritoneal cavity and along the intestine to avoid puncturing the tissue. After surgical recovery, metastases progression or regression was followed by non-invasive bioluminescence imaging twice a week until mice were sacrificed for ethical considerations (more than 20\% weight loss or prostration).

\subsection{Statistical analyzes}

Statistical analyzes (Kruskal-Wallis, Mann Whitney, 2 ways ANOVA, Mantel Cox) were performed using Graphpad prism® (La Jolla, California, USA).

\section{Results}

\subsection{Animal model characterization by in vivo multimodal imaging}

In vivo bioluminescence and bimodal tomographic imaging (combining 3D fluorescence imaging and microCT) were used to monitor primary tumor growth and metastasis (Fig. 3A and B). The use of non-invasive molecular imaging allowed individual longitudinal follow-up of tumor development in each animal and enabled an equal distribution of the mice within the three experimental groups before treatment (Fig. 3C). Multimodal imaging provided preoperative mol-

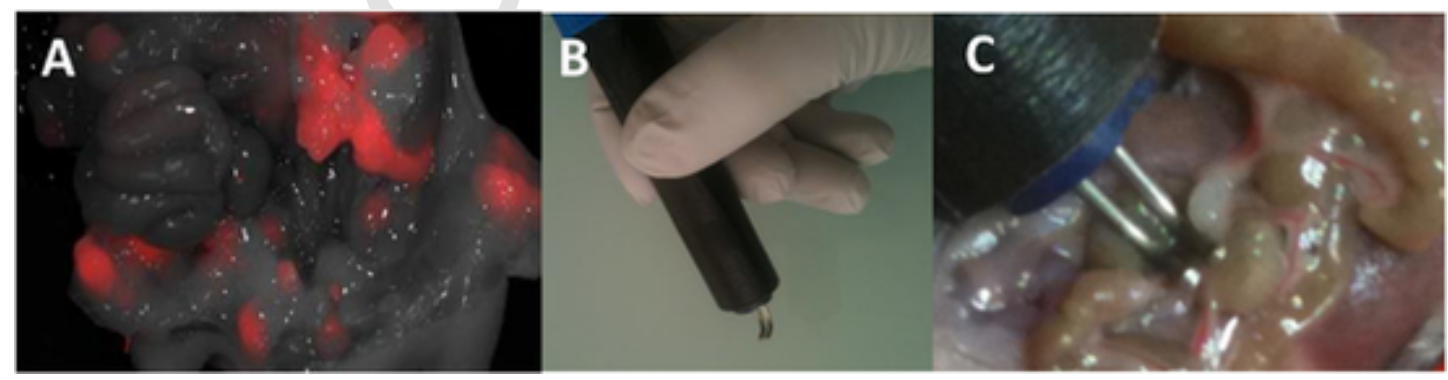

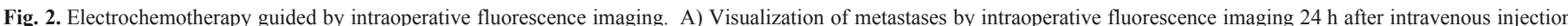

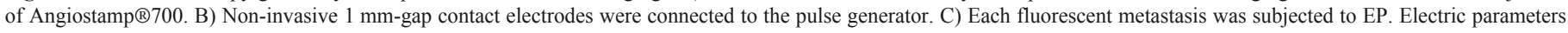
were 8 pulses $(100 \mu$ s at $10 \mathrm{~Hz}$ frequency). The amplitude was 110 or $130 \mathrm{~V}$. 

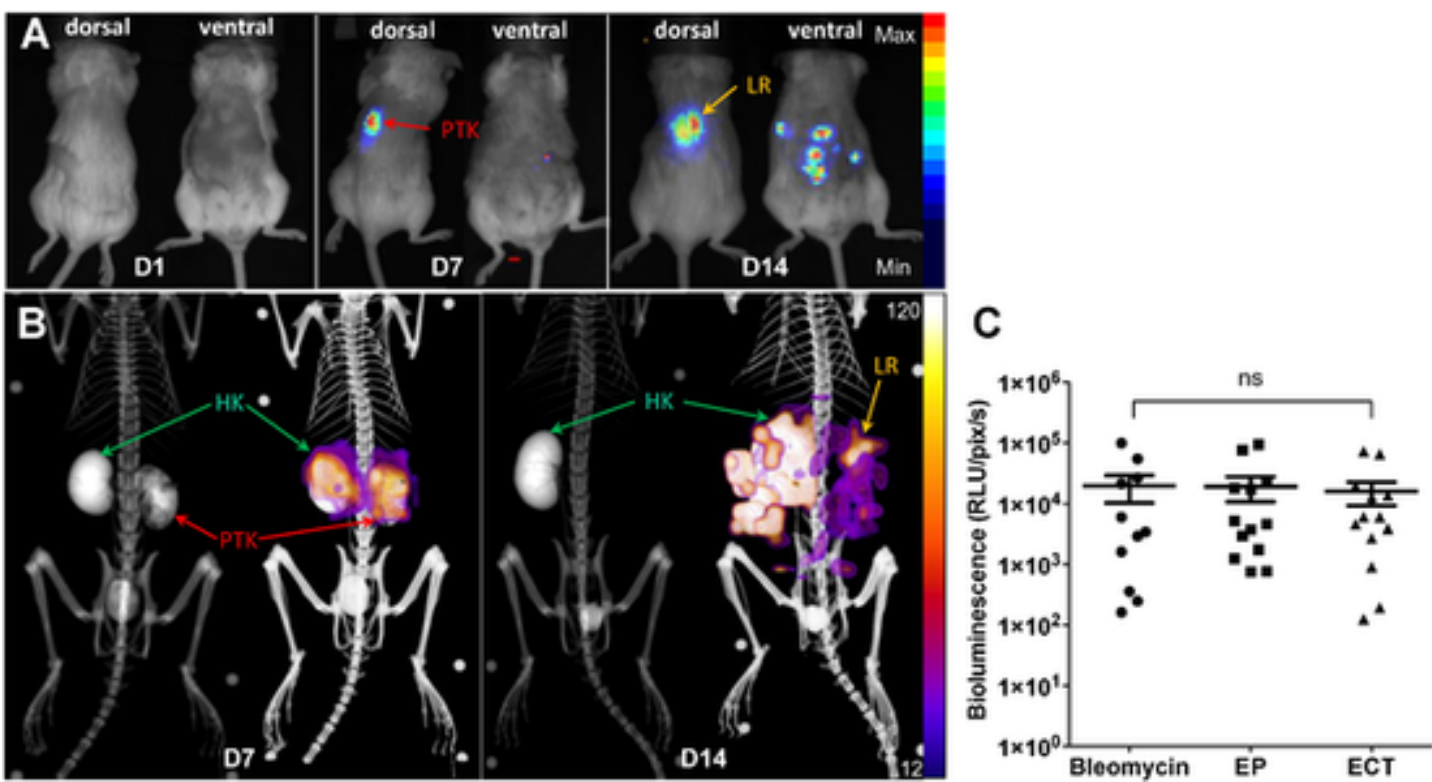

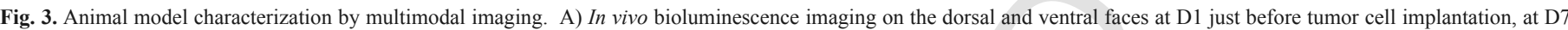

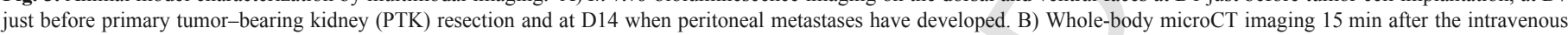

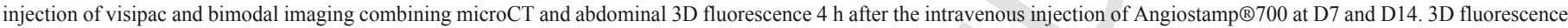

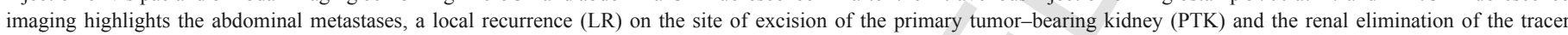

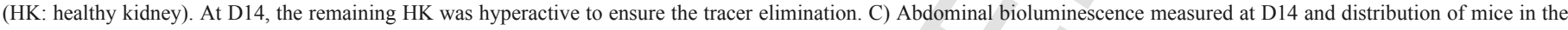

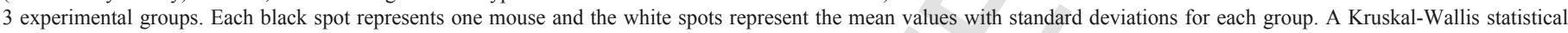
analysis was performed.

ecular diagnosis and accurate characterization of the animal model. Abdominal micro-metastases could be spotted before surgery and were then easily located during the surgery using intraoperative real time NIR fluorescence imaging (Fig. 2A). IF imaging was used to guide the application of the image-guided ECT protocol (Fig. 2C). Upon the completion of surgery, the progression of the residual nodules was measured using non-invasive bioluminescence over time. Because each animal constituted its own control, the inter-animal heterogeneity of the tumor growth was minimized.

\subsection{Effect of EP and ECT treatments on tumor burden and survival}

The average number of treated nodules per mouse was $14.2 \pm 3.5$ for EP and $14.4 \pm 2.9$ for ECT. Immediately after EP (with or without bleomycin), we observed that all pulsed nodules turned white. This phenomenon, which has been previously described, is due to a transient vascular lock, induced by EP, that temporary stops the blood flow [22]. Mouse recovery after surgery and EP or ECT treatment was good, with less than $10 \%$ mortality.

After the treatment, mice from the bleomycin group quickly presented severe metastatic progression as seen by bioluminescence imaging (Fig. 4A). This finding is consistent with the fact that a sub-optimal bleomycin concentration was used as compared to standard bleomycin chemotherapy protocols [14,15]. Indeed, 3 days after the bleomycin treatment (at day 18), the tumor burden had already reached $372 \%$ of the measure at the day before treatment (day 14). At the same time, the metastatic invasion was only mildly slowed in the EP group (203\% of the measure at day 14). In contrast, we observed a strong tumor growth inhibition within the ECT group, represented by a tumor burden at day 18 of $109 \%$ of the measure at day 14 (Fig. 4B). These differences were strongly amplified over time (Fig. 4C) and the reduced metastatic load in the ECT group was associated with a significantly increased survival rate (median lifespan: 34 days vs 25 days; ****p \$_amp_\$1t; 0.0001) while there was no significant difference in life span between the mice treated with bleomycin alone and the non-operated mice (median lifespan: 27 days vs 25 days, respectively) (Fig. 4D).

\subsection{Effect of the voltage intensity}

We then investigated whether increasing the voltage intensity from $110 \mathrm{~V}$ to $130 \mathrm{~V}$ could improve the therapeutic effect. For both EP and ECT, we observed a two fold reduction in the tumor burden when the voltage intensity was $130 \mathrm{~V}$ as compared to $110 \mathrm{~V}$ (Fig. 5A). However, there was a sharp deterioration in the survival rate of the mice treated with ECT at $130 \mathrm{~V}$ in comparison with ECT at $110 \mathrm{~V} \mathrm{(27} \mathrm{days}$ $v s .34$ days), indicating a global toxicity for this higher-intensity condition (Fig. 5B).

\section{Discussion}

In this study, we developed a syngeneic immuno-competent murine model in which micro-metastases appear secondarily to primary tumor growth and resection (Fig. 1). Primary tumor development and metastases invasion were finely characterized by non-invasive multimodal imaging combining i) bioluminescence to monitor tumor progression and ii) fluorescence tomography/microCT, using a tumor-targeted probe, to allow pre- and intraoperative molecular imaging (Fig. 3). Non-invasive imaging techniques facilitate the accurate diagnosis, staging and visualization of tumors, which are prerequisites for successful surgical therapy. Moreover, the use of tumor-specific labeling allows direct translation from preoperative diagnostic to image-guided surgery. Intraoperative real time visualization of the tumor, by highlighting the molecular signature obtained from preoperative imaging, allows the surgeon to be precisely guided within the cancerous tissues (Fig. 2). Until now, many different molecular imaging modalities, either alone or in combination with a navigation system, have been used to improve surgery. Those that provide 
A
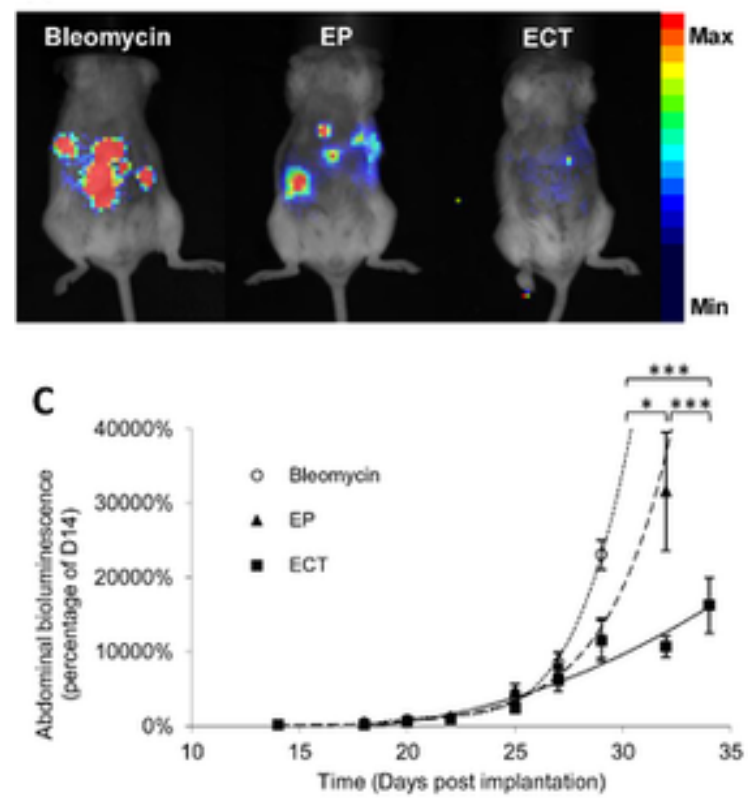

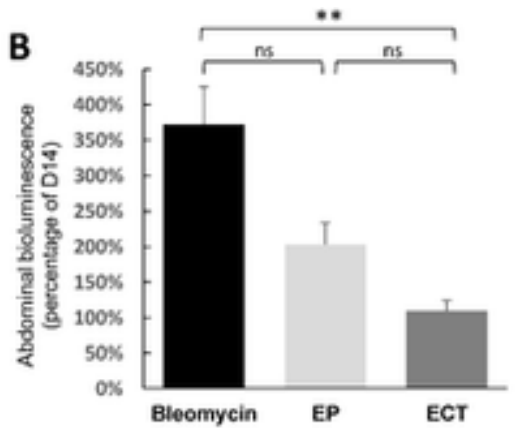

D

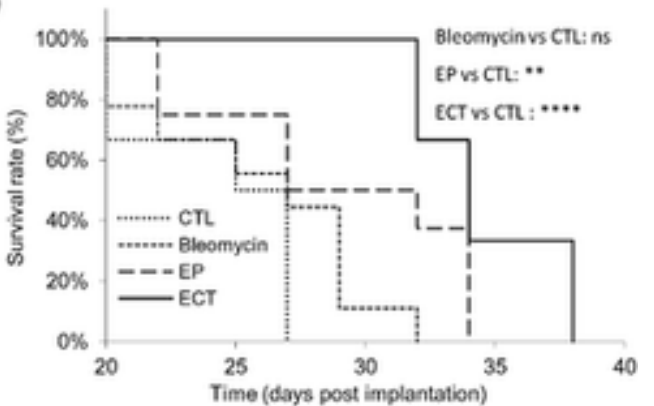

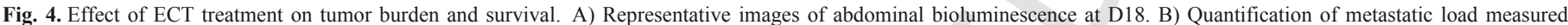

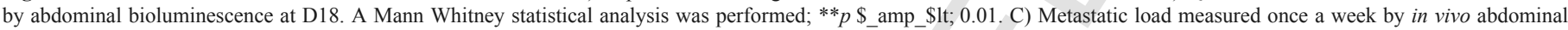

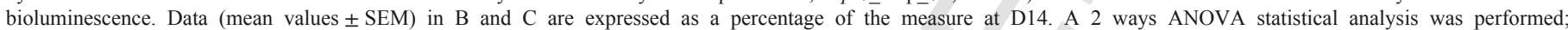

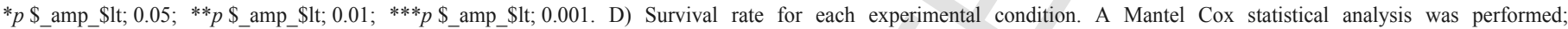
$* * p$ \$amp_\$lt; $0.01 ; * * * * p \$$ _amp_\$lt; 0.0001 .
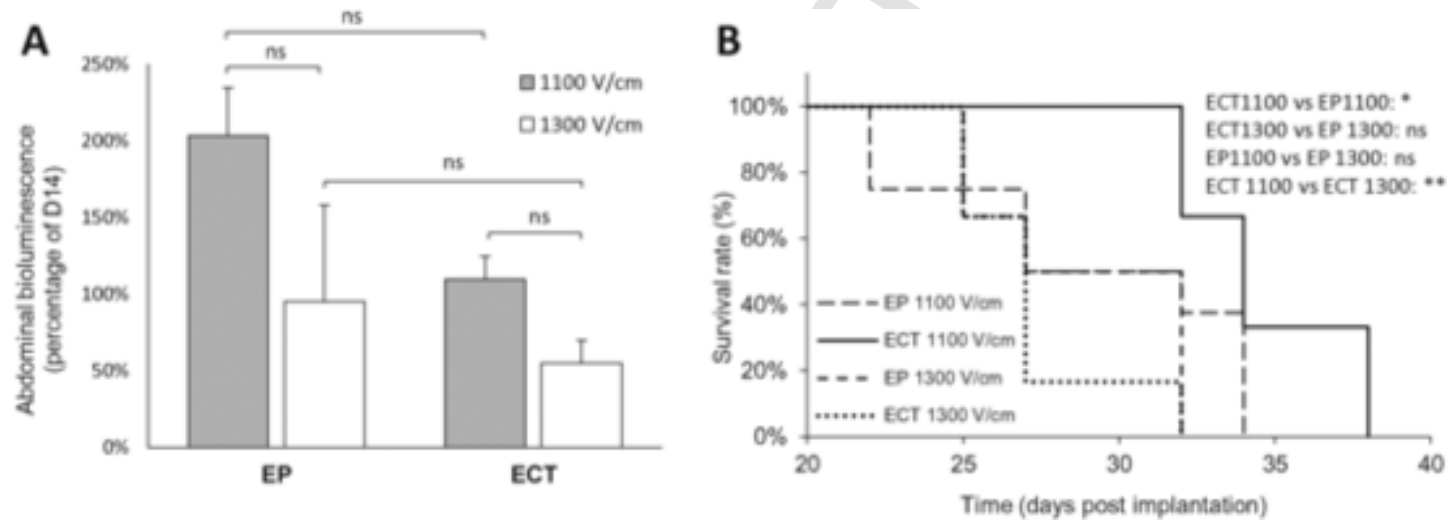

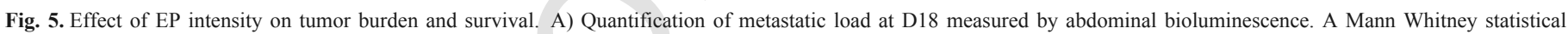
analysis was performed. B) Survival rate for each experimental condition. A Mantel Cox statistical analysis was performed; ${ }^{*} p \$ \_$amp_\$lt; $0.05 ; * * p \$ \_$amp_\$lt; 0.01 .

intraoperative guidance for the tumor resection, such as intraoperative nuclear imaging [23], Magnetic Resonance Imaging (MRI) [24,25], Ultra Sounds (US) [26], and fluorescence imaging, are important innovative tools, each with its own merits and limitations [7]. Among them, fluorescence imaging seems to be the most suitable for intraoperative surgical guidance because i) it uses non-ionizing photons, ii) it presents high sensitivity and resolution at low depth, iii) it offers a wide field of view compatible with the operative field, iv) it displays a high speed readout allowing real time imaging and v), the absence of radioactive products in the operating room is also contributing to make it the optimal choice for intraoperative guidance [27].

In addition to the choice of the imaging modality, another issue concerns the use of a relevant molecular imaging probe. Currently, the most promising fluorescent probes for cancer imaging are based on the active targeting of tumor-specific biomarkers. Indeed they offer better tumor to healthy tissue contrast than non-targeting agents that accumulate in the tumor passively via the enhanced permeability and retention (EPR) effect [28]. Currently, the translation of these fluorescent targeting probes to the clinic is still a major bottleneck that slows down the acceptance of optical imaging in human trials [29]. Various cancer biomarkers that are associated with malignant phenotypes, such as growth factor receptors, matrix metallo-proteases and other markers of angiogenesis, have been considered for the design of tumor-targeting agents. Most of these biomarkers, especially those located on tumor cell-surface, are suitable for active targeting. Thus, possible targets include the folate receptor, VEGF, EGFR, HER2, epCAM, PSMA and $\alpha v \beta 3$ integrin.

In this study, we focused on the $\alpha v \beta 3$ integrin, which is overexpressed during angiogenesis and in aggressive tumor cells [30-32]. 
RGD peptides have been identified as high affinity ligands for the $\alpha v \beta 3$ integrin and the preclinical applications of fluorescent RGD peptides in targeted surgery have been reported in ovarian cancer [33] and colorectal intra-abdominal metastases [34]. Our group previously developed a fluorescent molecular scaffold presenting four copies of a cRGD called Angiostamp ${ }^{\mathrm{TM}}$. We demonstrated the high affinity and specificity of Angiostamp ${ }^{\mathrm{TM}}$ for the $\alpha \mathrm{v} \beta 3$ integrin, both in vitro and in vivo, in various animal models including peritoneal carcinomatosis and ovarian metastasis in mice [32,33], orthotopic head and neck squamous cell carcinoma in mice [5], osteosarcoma in rats [34], and fibrosarcoma in cats [4]. In each of these diverse conditions, Angiostamp ${ }^{\mathrm{TM}}$ displayed a high signal in tumor tissue with low nonspecific background, generating a very good tumor contrast that allowed visualization and precise delineation of tumor margins and significantly improved the surgical resection. Moreover, the surgery duration was significantly reduced, which may also have an important impact on the patient outcome [3].

In the very invasive metastatic model used in this study, surgical resection of spotted micro-metastases remained a challenge because of the potential excision-related damage that can occur on blood vessels or the intestine wall, leading to hemorrhage or septicemia respectively. Furthermore, our previous results demonstrated that the use of IF imaging led to the identification of two times more tumor nodules than the surgeon's eyes can distinguish, and particularly some very small ones (down to $\sim 250$ cells) [3]. Because of its small size, the mouse is a very tricky model to perform resection surgery. Thus, improving the visualization of micro-nodules, by increasing the number of resection gestures also increases the risk of damage. Indeed, in our mouse model, the post-operative morbidity was very high in the context of an exhaustive surgical resection of the nodules (up to $85 \%$ of operative or post-operative mortality, data not shown). In the clinical situation, the surgeon will advisedly leave some tumor nodules that cannot be resected because of their delicate location and will rely on intracavital hyperthermic chemotherapy to treat nodules that are smaller than $2.5 \mathrm{~mm}$. Nonetheless, if bigger nodules are left they will certainly contribute to relapse. Therefore, there is a need for improved loco-regional treatments for use in combination with surgery [8].

In this study we evaluated a new therapeutic approach combining intraoperative image-guided tumor location and ECT, using a peritoneal micro-metastases model. No resection surgery was performed, but nodules identified by IF were submitted to EP following intravenous injection of bleomycin. Under these conditions fewer than $10 \%$ of the mice died following surgeries for EP or ECT treatment.

The main mechanism of the antitumor effect of ECT is the enhanced delivery of cytotoxic molecules into tumor cells $[12,18]$. A suitable drug for ECT treatment is a hydrophilic molecule that cannot easily cross the intact plasma membrane of the cell. With ECT, a high transport across the membrane is obtained for these hydrophilic molecules. Therefore low concentrations can be administered to achieve intracellular concentrations suitable to induce efficient cytotoxic activities, thus avoiding deleterious side effects. Bleomycin and cisplatin are the two chemotherapeutic drugs currently used in the standardized ECT protocols in clinic. Bleomycin is a non-permeant antineoplastic antibiotic isolated from Streptomyces verticillus [35]. Its cytotoxicity results from its ability to make single and double strand DNA breaks in mammalian cells [36]. Cisplatin is a low-permeant platinum-based antineoplastic agent [37]. Its cytotoxic mode of action is mediated by its interaction with DNA to form DNA adducts, inducing intra-strand cross-links. However, due to their relative hydrophilicity, these compounds have a very low cellular uptake $[10,13]$. The hampered cellular transmembrane transport of these molecules can be overcome by EP. The effectiveness of bleomycin and cisplatin in ECT has been demonstrated in preclinical studies using several tumor models but also for different cancer types in human and equine clinical trials $[10,13]$. ECT is highly effective, with complete response rates between 60 and $70 \%$ and objective response rate around $80 \%[10,18,19]$. ECT has already been shown to be efficient for deep tumors $[9,38,39]$. In the case of peritoneal metastases, we believe that intraoperative image-guided tumor localization can increase the effectiveness of the ECT approach. Indeed, in our study we demonstrated the success of ECT treatment to slow down metastases progression and to increase the survival rate in a very aggressive peritoneal metastases model without resorting to tumor removal.

Beyond the cytotoxic action of bleomycin, additional mechanisms may contribute to the success of this therapy. One such mechanism involves the vascular disrupting action of ECT. While electroporation alone reduces blood flow, ECT has a delayed killing effect on tumor vasculature endothelial cells, leading to prolonged reduction of tumor blood flow and consequently to tumor hypoxia and eventually to tumor necrosis [40]. In the present study, we observed that the treated metastatic nodules became white immediately after EP or ECT. This phenomenon is due to the transient vascular lock after EP and to a more prolonged blood flow reduction when combined with the killing effect on vascular endothelial cells in presence of bleomycin [41].

It is known that the local field strength in the tissue must be above a critical value for ECT to be effective. This local field value is under the control of the applied voltage and the geometry of the electrodes $[42,43]$. When using higher electric voltage $(130 \mathrm{~V})$, the overall survival of the animals was not statistically improved by ECT as compared to EP alone. Indeed, when a tissue is submitted to high voltage, irreversible electroporation (IRE) may occur [44-46]. In IRE, the application of high voltage across the tissue alters the transmembrane potential and disrupts the lipid bilayer of cells, allowing the exchange of intra and extra-cellular components that can contribute to cell death. Tissues under IRE ablation showed a peak apoptotic rate at $24 \mathrm{~h}$ after IRE ablation with viable tissues at the peripheral rim of treated tissues in histological analysis on prostate tumors [47]. The cell destruction mechanism of IRE is not based on thermal damage. IRE was not studied until the early 2000s in the context of medical applications as an ablative method but now several surgical applications are under clinical trials [45]. In our study, we hypothesized that EP (130V) could also induce cell mortality by an IRE like process.

Another mechanism involving the immune system has been described to explain the overall efficacy of ECT. Indeed, when ECT with bleomycin or cisplatin have been applied to subcutaneous transplanted tumors in nude or immune-competent mice, the ECT treatment was less effective in nude mice, thus indicating a role for the immune system in clearing tumors $[20,48]$. However, it is not yet fully understood how ECT may trigger the immune system $[49,50]$. In our experiments, we used immuno-competent BALB/c mice, bearing a very aggressive tumor model that kills non-treated mice within 20 to 27 days. Due to this aggressivity, the delay between the ECT treatment and the death of the mice might not be long enough to promote a strong and efficient stimulation of the immune system and it is thus unlikely that the immune reaction participated to the antitumor effect. Indeed, we did not observe a complete regression of the disease but ECT led to a significant $(* * * * p \$$ \$amp_\$1t; 0.0001) $36 \%$ increase in median lifespan (corresponding to approximately 12 days). It would be interesting to evaluate the presence and intensity of the ECT-primed antitumor immune response, but this would necessitate the use of a more adapted animal model. Finally, it 
has been shown that ECT can be combined with another therapeutic approach such as irradiation [51]. Thus ECT guided by intraoperative fluorescence imaging could be an additional tool at the disposition of clinicians for improving the outcome of such patients.

\section{Conclusion}

In this study, we used a very aggressive peritoneal micro-metastases model that we finely characterized using non-invasive multimodal imaging. The use of tumor-specific fluorescent labeling allowed direct translation from preoperative molecular imaging and diagnosis to intraoperative visualization of the tumor nodules. We implemented a new therapeutic approach using tumor-targeted fluorescence guided ECT which efficiently minimized collateral damage related to resection surgery and led to a significant increase in lifespan. ECT has already been approved in several EU countries (Denmark, Germany, Greece, Italy, Poland, Portugal, Slovenia, and the UK) and we believe that intraoperative image-guided tumor localization can increase the effectiveness of this approach in treating peritoneal metastases.

\section{Acknowledgements}

Research was conducted within the scope of the EBAM European Associated Laboratory (LEA) and network of the COST action TD1104. Optical systems were acquired thanks to France Life Imaging (FLI, French program "Investissement d'Avenir"; grant "Infrastructure d'avenir en Biologie Santé", ANR-11-INBS-0006). Proof Reading Services corrected the manuscript.

\section{References}

[1] R.L. Harmon, P.H. Sugarbaker, Prognostic indicators in peritoneal carcinomatosis from gastrointestinal cancer, Int. Semin. Surg. Oncol. 2-3 (2005).

[2] M. Gutowski, M. Carcenac, D. Pourquier, C. Larroque, B. Saint-Aubert, P. Rouanet, A. Pèlegrin, Intraoperative immunophotodetection for radical resection of cancers: evaluation in an experimental model, Clin. Cancer Res. 7 (2001) 1142-1148.

[3] M. Keramidas, V. Josserand, C. Righini, C. Wenk, C. Faure, D. Boturyn, P. Rizo, P. Dumy, J.L. Coll, Intraoperative near-infrared image guided surgery of peritoneal carcinomatosis in a preclinical mouse model, Br. J. Surg. 97 (2010) 737-743.

[4] C. Wenk, V. Josserand, S. Guillermet, C. Tenaud, D. Watrelot-Virieux, C. Carozzo, F. Ponce, J.L. Coll, Near-infrared optical guided surgery of highly infiltrative fibrosarcomas in cats using an anti-avß3 integrin molecular probe, Cancer Lett. 334 (2013) 188-195.

[5] I. Atallah, C. Milet, J.L. Coll, E. Reyt, C.A. Righini, A. Hurbin, Role of near-infrared fluorescence imaging in head and neck cancer surgery: from animal models to humans, Eur. Arch. Otorhinolaryngol. 272 (2015) 2593-2600.

[6] L. Bu, B. Shen, Z. Cheng, Fluorescent imaging of cancerous tissues for targeted surgery, Adv. Drug Deliv. Rev. 76 (2014) 21-38.

[7] Hussain, Q.T. Nguyen, Molecular imaging for cancer diagnosis and surgery, Adv. Drug Deliv. Rev. 66C (2014) 90-100.

[8] P.D. Hansen, M.A. Cassera, R.F. Wolf, Ablative technologies for hepatocellular, cholangiocarcinoma, and metastatic colorectal cancer of the liver, Surg. Oncol. Clin. N. Am. 97-119 (2015)

[9] D. Miklavčič, G. Serša, E. Brecelj, J. Gehl, D. Soden, G. Bianchi, P. Ruggieri, C.R. Rossi, L.G. Campana, T. Jarm, Electrochemotherapy: technological advancements for efficient electroporation-based treatment of internal tumors, Med. Biol. Eng. Comput. 50 (2012) 1213-1225.

[10] B. Mali, T. Jarm, M. Snoj, G. Sersa, D. Miklavcic, Antitumor effectiveness of electrochemotherapy: a systematic review and meta-analysis, Eur. J. Surg. Oncol. 39 (2013) 4-16.

[11] L.M. Mir, S. Orlowski, J. Belehradek Jr., C. Paoletti, Electrochemotherapy potentiation of antitumour effect of bleomycin by local electric pulses, Eur. J. Cancer 27 (1991) 68-72.

[12] G. Sersa, D. Miklavcic, M. Cemazar, Z. Rudolf, G. Pucihar, M. Snoj, Electrochemotherapy in treatment of tumours, Eur. J. Surg. Oncol. 34 (2008) 232-240.
[13] Y. Tamzali, L. Borde, M.P. Rols, M. Golzio, F. Lyazrhi, J. Teissie, Successful treatment of equine sarcoids with cisplatin electrochemotherapy: a retrospective study of 48 cases, Equine Vet. J. 44 (2012) 214-220.

[14] L.M. Mir, M. Belehradek, C. Domenge, S. Orlowski, B. Poddevin, J. Belehradek Jr., G. Schwaab, B. Luboinski, C. Paoletti, Electrochemotherapy, a new antitumor treatment: first clinical trial, C. R. Acad. Sci. III 313 (1991) 613-618.

[15] M. Belehradek, C. Domenge, B. Luboinski, S. Orlowski, J. Belehradek Jr., L.M. Mir, Electrochemotherapy, a new antitumor treatment. First clinical phase I-II trial, Cancer 72 (1993) 3694-3700.

[16] V. Mlakar, V. Todorovic, M. Cemazar, D. Glavac, G. Sersa, Electric pulses used in electrochemotherapy and electrogene therapy do not significantly change the expression profile of genes involved in the development of cancer in malignant melanoma cells, BMC Cancer 9 (2009) 299.

[17] V. Todorovic, G. Sersa, V. Mlakar, D. Glavac, M. Cemazar, Assessment of the tumourigenic and metastatic properties of SK-MEL28 melanoma cells surviving electrochemotherapy with bleomycin, Radiol. Oncol. 46 (2012) 32-45.

[18] C. Cabula, L.G. Campana, G. Grilz, S. Galuppo, R. Bussone, L. De Meo, A. Bonadies, P. Curatolo, M. De Laurentiis, M. Renne, S. Valpione, T. Fabrizio, N. Solari, M. Guida, A. Santoriello, M. D'Aiuto, R. Agresti, Electrochemotherapy in the treatment of cutaneous metastases from breast cancer: a multicenter cohort analysis, Ann. Surg. Oncol. 3 (2015) 442-450.

[19] R. Cadossi, M. Ronchetti, M. Cadossi, Locally enhanced chemotherapy by electroporation: clinical experiences and perspective of use of electrochemotherapy, Future Oncol. 10 (2014) 877-890.

[20] G. Sersa, D. Miklavcic, M. Cemazar, J. Belehradek, T. Jarm, L.M. Mir, Electrochemotherapy with CDDP on LPB sarcoma: comparison of the anti-tumor effectiveness in immunocompetent and immunodeficient mice, Bioelectrochem. Bioenerg. 43 (1997) 279-283.

[21] A. Koenig, L. Hervé, G. Gonon, V. Josserand, M. Berger, J.M. Dinten, J. Boutet, P. Peltié, J.L. Coll, P. Rizo, Fluorescence diffuse optical tomography for free-space and multifluorophore studies, J. Biomed. Opt. 15 (2010) 016016.

[22] J. Gehl, T. Skovsgaard, L.M. Mir, Vascular reactions to in vivo electroporation: characterization and consequences for drug and gene delivery, Biochim. Biophys. Acta 1569 (2002) 51-58.

[23] N.C. Hall, S.P. Povoski, J. Zhang, M.V. Knopp, Martin EW use of intraoperative nuclear medicine imaging technology: strategy for improved patient management, Expert Rev. Med. Devices 10 (2013) 149-152.

[24] W.A. Hall, C.L. Truwit, Intraoperative MR imaging, Magn. Reson. Imaging Clin. N. Am. 13 (2005) 533-543.

[25] P.M. Black, T. Moriarty, E. Alexander III, P. Stieg, E.J. Woodard, P.L. Gleason, C.H. Martin, R. Kikinis, R.B. Schwartz, F.A. Jolesz, Development and implementation of intraoperative magnetic resonance imaging and its neurosurgical applications, Neurosurgery 41 (1997) 831-842.

[26] R.A. Kane, Intraoperative ultrasonography: history, current state of the art, and future directions, J. Ultrasound Med. 23 (2004) 1407-1420.

[27] N.J. Harlaar, W. Kelder, A. Sarantopoulos, J. Bart, G. Themelis, G.M. van Dam, V. Ntziachristos, Real-time near infrared fluorescence (NIRF) intra-operative imaging in ovarian cancer using an alpha(v)beta(3-)integrin targeted agent, Gynecol. Oncol. 128 (2013) 590-595.

[28] M. Yu, J. Zheng, Clearance pathways and tumor targeting of imaging nanoparticles, ACS Nano 9 (2015) 6655-6674.

[29] E.L. Rosenthal, J.M. Warram, K.I. Bland, K.R. Zinn, The status of contemporary image guided modalities in oncologic surgery, Ann. Surg. 261 (2015) 46-55.

[30] J.D. Hood, D.A. Cheresh, Role of integrins in cell invasion and migration, Nat Rev. Cancer 2 (2002) 91-100.

[31] M. Hutteman, J.S. Mieog, J.R. van der Vorst, J. Dijkstra, P.J. Kuppen, A.M. van der Laan, H.J. Tanke, E.L. Kaijzel, I. Que, C.J. van de Velde, C.W. Lowik, A.L. Vahrmeijer, Intraoperative near-infrared fluorescence imaging of colorectal metastases targeting integrin alpha(v)beta(3) expression in a syngeneic rat model, Eur. J. Surg. Oncol. 37 (2011) 252-257.

[32] Z.H. Jin, V. Josserand, J. Razkin, E. Garanger, D. Boturyn, M.C. Favrot, P. Dumy, J.L. Coll, Noninvasive optical imaging of ovarian metastases using Cy5-labeled RAFT-c(-RGDfK-)4, Mol. Imaging 5 (2006) 188-197.

[33] E. Mery, E. Jouve, S. Guillermet, M. Bourgognon, M. Castells, M. Golzio, P. Rizo, J.P. Delord, D. Querleu, B. Couderc, Intraoperative fluorescence imaging of peritoneal dissemination of ovarian carcinomas. A preclinical study, Gynecol. Oncol. 122 (2011) 155-162.

[34] A. Dutour, V. Josserand, D. Jury, S. Guillermet, A.V. Decouvelaere, F. Chotel, T. Pointecouteau, P. Rizo, J.L. Coll, J.Y. Blay, Targeted imaging of $\alpha(v) \beta(3)$ expressing sarcoma tumor cells in vivo in pre-operative setting using near infrared: a potential tool to reduce incomplete surgical resection, Bone 62 (2014) 71-78.

[35] L.M. Mir, O. Tounekti, S. Orlowski, Bleomycin: revival of an old drug, Gen. Pharmacol. 27 (1996) 745-748. 
[36] O. Tounekti, A. Kenani, N. Foray, S. Orlowski, L.M. Mir, The ratio of singleto double-strand DNA breaks and their absolute values determine cell death pathway, Br. J. Cancer 84 (2001) 1272-1279.

[37] Z.H. Siddik, Cisplatin: mode of cytotoxic action and molecular basis of resistance, Oncogene 22 (2003) 7265-7279.

[38] I. Edhemovic, E.M. Gadzijev, E. Brecelj, D. Miklavcic, B. Kos, A. Zupanic, B. Mali, T. Jarm, D. Pavliha, M. Marcan, G. Gasljevic, V. Gorjup, M. Music, T.P. Vavpotic, M. Cemazar, M. Snoj, G. Sersa, Electrochemotherapy: a new technological approach in treatment of metastases in the liver, Technol. Cancer Res. Treat. 10 (2011) 475-485.

[39] R. Girelli, S. Prejanò, I. Cataldo, V. Corbo, L. Martini, A. Scarpa, B. Claudio, Feasibility and safety of electrochemotherapy (ECT) in the pancreas: a pre-clinical investigation, Radiol. Oncol. 49 (2015) 147-154.

[40] G. Sersa, T. Jarm, T. Kotnik, A. Coer, M. Podkrajsek, M. Sentjurc, D. Miklavcic, M. Kadivec, S. Kranjc, A. Secerov, M. Cemazar, Vascular disrupting action of electroporation and electrochemotherapy with bleomycin in murine sarcoma, Br. J. Cancer 98 (2008) 388-398.

[41] B. Markelc, G. Sersa, M. Cemazar, Differential mechanisms associated with vascular disrupting action of electrochemotherapy: intravital microscopy on the level of single normal and tumor blood vessels, PLoS One 8 (2013), e59557.

[42] S. Corovic, I. Lackovic, P. Sustaric, T. Sustar, T. Rodic, D. Miklavcic, Modeling of electric field distribution in tissues during electroporation, Biomed. Eng. Online 12 (2013) 12-16.

[43] M. Marčan, D. Pavliha, B. Kos, T. Forjanič, D. Miklavčič, Web-based tool for visualization of electric field distribution in deep-seated body structures and planning of electroporation-based treatments, Biomed. Eng. Online 14 (2015) S3-S4.
[44] L. Horowitz, R.V. Davalos, L.M. Mir, B. Rubinsky, In vivo results of a new focal tissue ablation technique: irreversible electroporation, IEEE Trans. Biomed. Eng. 53 (2006) 1409-1415.

[45] B. Rubinsky, Irreversible electroporation in medicine, Technol. Cancer Res. Treat. 6 (2007) 255-260.

[46] M.A. Phillips, R. Narayan, T. Padath, B. Rubinsky, Irreversible electroporation on the small intestine, Br. J. Cancer 106 (2012) 490-495.

[47] H.B. Kim, C.K. Sung, K.Y. Baik, K.W. Moon, H.S. Kim, J.H. Yi, J.H. Jung, M.H. Moon, Choi OK changes of apoptosis in tumor tissues with time after irreversible electroporation, Biochem. Biophys. Res. Commun. 435 (2013) $651-656$.

[48] A. Sedlar, T. Dolinsek, B. Markelc, L. Prosen, S. Kranjc, M. Bosnjak, T. Blagus, M. Cemazar, G. Sersa, Potentiation of electrochemotherapy by intramuscular IL-12 gene electrotransfer in murine sarcoma and carcinoma with different immunogenicity, Radiol. Oncol. 46 (2012) 302-311.

[49] C.Y. Calvet, D. Famin, F.M. André, L.M. Mir, Electrochemotherapy with bleomycin induces hallmarks of immunogenic cell death in murine colon cancer cells, Oncoimmunology (2014), e28131.

[50] N. Mozzillo, E. Simeone, L. Benedetto, M. Curvietto, D. Giannarelli, G. Gentilcore, R. Camerlingo, M. Capone, G. Madonna, L. Festino, C. Caracò, G. Di Monta, U. Marone, M. Di Marzo, A.M. Grimaldi, S. Mori, G. Ciliberto, P.A. Ascierto, Assessing a novel immuno-oncology-based combination therapy: Ipilimumab plus electrochemotherapy, Oncoimmunology 4 (2015), e1008842.

[51] S. Kranjc, M. Cemazar, A. Grosel, M. Sentjurc, G. Sersa, Radiosensitising effect of electrochemotherapy with bleomycin in LPB sarcoma cells and tumors in mice, BMC Cancer 5 (2005) 115. 